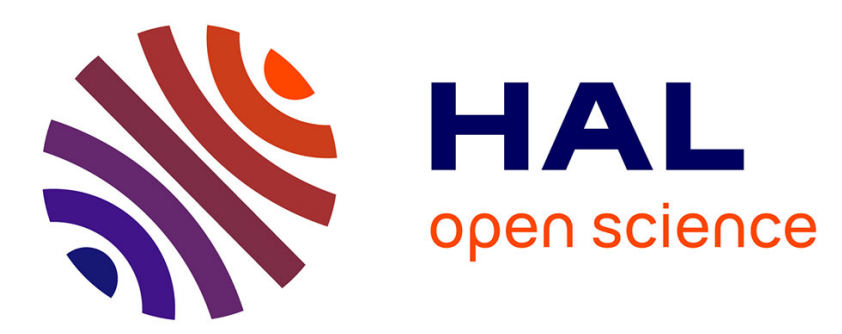

\title{
Thruster's dead-zones compensation for the actuation system of an underwater vehicle
}

Benoît Ropars, Adrien Lasbouygues, Lionel Lapierre, David Andreu

\section{To cite this version:}

Benoît Ropars, Adrien Lasbouygues, Lionel Lapierre, David Andreu. Thruster's dead-zones compensation for the actuation system of an underwater vehicle. ECC: European Control Conference, Jul 2015, Linz, Austria. pp.741-746, 10.1109/ECC.2015.7330631 . lirmm-01310931

\section{HAL Id: lirmm-01310931 https://hal-lirmm.ccsd.cnrs.fr/lirmm-01310931}

Submitted on 3 May 2016

HAL is a multi-disciplinary open access archive for the deposit and dissemination of scientific research documents, whether they are published or not. The documents may come from teaching and research institutions in France or abroad, or from public or private research centers.
L'archive ouverte pluridisciplinaire HAL, est destinée au dépôt et à la diffusion de documents scientifiques de niveau recherche, publiés ou non, émanant des établissements d'enseignement et de recherche français ou étrangers, des laboratoires publics ou privés. 


\title{
Thruster's Dead-zones Compensation for the Actuation System of an Underwater Vehicle
}

\author{
B. Ropars ${ }^{\circ *}$, A. Lasbouygues*, L. Lapierre* and D. Andreu*
}

\begin{abstract}
This paper addresses a control allocation method in order to compensate for the effect of thrusters' dead-zones, on the actuation system of an underwater vehicle. This solution concurrently considers dead-zone effect and actuation limitation, in order to increase system reactivity. Moreover the impact on energy consumption is also considered.

Experimental results are given to demonstrate the effectiveness and correctness of the proposed method.
\end{abstract}

\section{INTRODUCTION}

The last decade has witnessed an increasing interest for underwater robotics, with audacious applications that require pushing further the need for original and efficient solutions, in terms of robustness, reactivity and dependability. The design of the actuation system, and the management of its redundancy, is a current major issue. Indeed, a large number of underwater vehicles have more thrusters than controllable degrees of freedom (DOF), resulting in an over-actuated system [1-5].

In [1-2], this structural redundancy is used to achieve dependability, providing fault tolerance to face the loss of a thruster. Optimization of thrust efficiency is also treated, with a heuristic approach in [8].

The structural redundancy of the system offers the possibility to compute the control allocation in order to realize different prioritized tasks. For example [4] uses a matrix of thrusters' readiness as a secondary function, as stated in [6]. [7] proposes a heuristic to optimize control performances, including energy consumption..

The management of actuation redundancy can also be performed by adding joint constraints on the control objective of redundant manipulators, using prioritized-tasks, as stated in [9-10].

In our case, we use the secondary task approach to add additional constraints to the thrust allocation of a horizontallyredundant underwater vehicle, in order to remove thrusters' dead-zone effect from the actuation response, respect actuator saturation and control system reactivity. Impact on energy consumption is considered. Its effect is minimized.

The paper is organized as follows. Chapter 2 states the problem, introduces notation and presents the system. Chapter 3 exposes our solution. Chapter 4 experimentally demonstrates its performance. Chapter 5 concludes the study.

\footnotetext{
*LIRMM, University Montpellier II, Montpellier, France, (\{name\}@lirmm.fr).

${ }^{\circ}$ Ciscrea, Toulon, France (bro@ciscrea.fr)
}

\section{PRoBlem STATEMENT}

This work and experiments are based on the Remotely Operated Vehicle (ROV), named Jack, manufactured by the Ciscrea Company ${ }^{1}$, cf. Fig 1 and 8. Let's first introduce the notation that will be used in the sequel.

\section{A. Notation}

Let $\{U\}$ be the universal coordinate frame and $\{B\}$ be the body frame. In the sequel, $\boldsymbol{\eta}_{\mathbf{U}}$ expresses the system state in $\{U\}, \mathbf{v}_{\mathbf{B}}$ is the system velocities in $\{B\}$ and $\mathbf{F}_{\mathbf{B}, \mathbf{6}}$ represents forces and torques provided by the actuation system w.r.t. $\{B\}$, as stated on Equations 1 and illustrated at Fig 1.

$$
\begin{gathered}
\boldsymbol{\eta}_{\mathbf{U}}=[x, y, z, \phi, \theta, \psi]^{T} \\
\boldsymbol{\eta}_{\mathbf{B}}=[u, v, w, p, q, r]^{T} \\
\mathbf{F}_{\mathbf{B}, \mathbf{6}}=\left[F_{u}, F_{v}, F_{w}, \Gamma_{p}, \Gamma_{q}, \Gamma_{r}\left[^{T}\right.\right.
\end{gathered}
$$

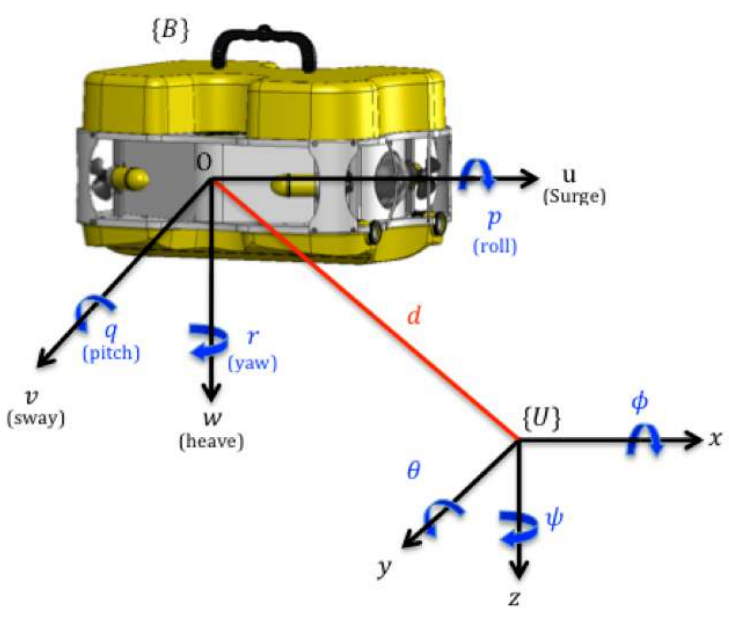

Figure 1. Frames definition, for Jack

\section{B. Actuation system architecture}

The Jack vehicle is equipped with 6 thrusters able to drive 5 degrees of freedom (DOF). The uncontrolled DOF, the pitch, is naturally stable considering mass and buoyancy restoring forces. Note that these restoring forces also induce a natural stabilization of the roll DOF.

In this study, we only consider the motion in the horizontal plane. 4 thrusters are used in this plane, thus providing

${ }^{1}$ www.ciscrea.net 
structural redundancy of the actuation system, as depicted in Figure 2.

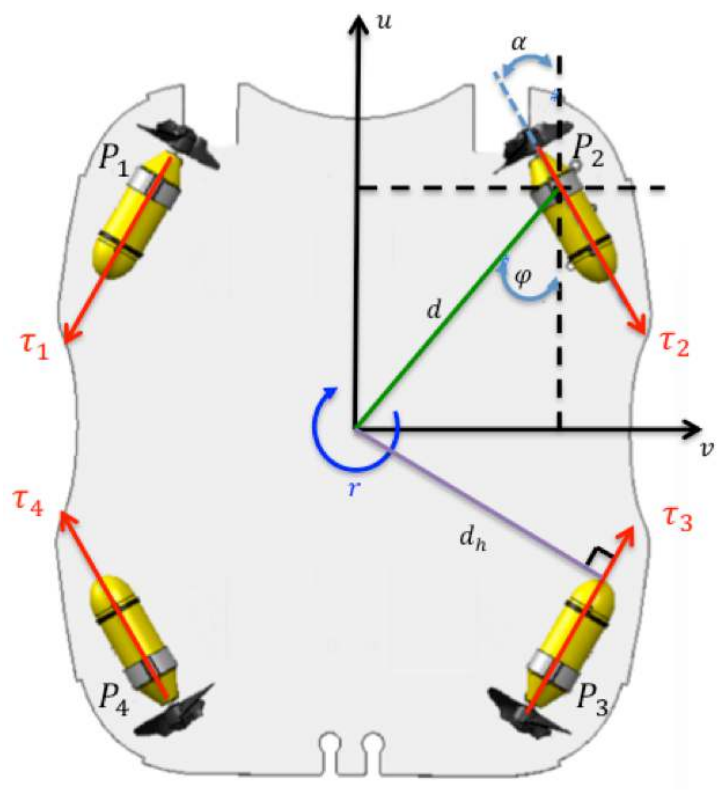

Figure 2. Position of horizontal thrusters

Each thruster is fixed on the robot on a point $P_{i}$, $i=1,2,3,4$, defined by angle $\varphi$ and distance $d$ w.r.t. $\{B\}$. Perpendicular distance to the thruster's direction is denoted $d_{h}$. Orientation of the produced thrust, denoted $F_{m, i}$, is defined with the angle $\alpha$, as depicted in Figure 2. Thrust is driven by an electric motor, with input $c_{m, i}$. The actuation system thus provides 4 forces $\mathbf{F}_{\mathbf{m}}=\left[F_{m, i}\right]^{T}$, with 4 motor inputs $\mathbf{c}_{\mathbf{m}}=\left[c_{m, i}\right]^{T}$. The resulting forces and torque expressed in the body frame is $\mathbf{F}_{\mathbf{B}}=\left[\begin{array}{lll}F_{u} & F_{v} & \Gamma_{r}\end{array}\right]^{T}$.

The 'vectorial' thrusters' configuration exhibits interesting properties, which will be used in the sequel: an identical command applied to the 4 thrusters induces a null resulting force and torque, as expressed in (8). Meanwhile, considering the thruster's position in $\{B\}$, we define the actuation model:

$$
\underbrace{\left[\begin{array}{l}
F_{u} \\
F_{v} \\
\Gamma_{r}
\end{array}\right]}_{\mathbf{F}_{\mathbf{B}}}=\underbrace{\left[\begin{array}{cccc}
-\cos \alpha & -\cos \alpha & \cos \alpha & \cos \alpha \\
-\sin \alpha & \sin \alpha & \sin \alpha & -\sin \alpha \\
-d_{h} & d_{h} & -d_{h} & -d_{h}
\end{array}\right]}_{\mathbf{M}_{\mathbf{G}}} \cdot \underbrace{\left[\begin{array}{l}
F_{m, 1} \\
F_{m, 2} \\
F_{m, 3} \\
F_{m, 4}
\end{array}\right]}_{\mathbf{F}_{\mathbf{m}}}
$$

Where $\mathbf{M}_{\mathbf{G}}$ expresses the geometrical distribution of thrusters, $\mathbf{F}_{\mathbf{B}}$ is the horizontal resulting forces and torque, expressed in $\{B\}$ and $\mathbf{F}_{\mathbf{m}}$ contains the forces produced by each of the 4 thrusters. The following equation expresses the actuation system model.

$$
\mathbf{F}_{\mathbf{B}}=\mathbf{M}_{\mathbf{G}} \cdot \mathbf{F}_{\mathbf{m}}
$$

\section{Redundancy management}

For control design purpose, the previous actuation model (3) has to be inverted. Indeed, the actuation demand, coming from a control law or the operator, will result in a set of desired forces and torque (denoted $\mathbf{F}_{\mathbf{B}}^{\mathbf{d}}$ ) to be applied by the actuation system. The inverse actuation model allows computing the desired thrusters' action according to the relation:

$$
F_{m}=M_{G}^{+} \cdot F_{B}^{d}
$$

Of course, since $\mathbf{M}_{\mathbf{G}}$ is not square and is not directly invertible. One can classically use the Moore-Penrose pseudo inversion if the matrix $\mathbf{M}_{\mathbf{G}} \cdot \mathbf{M}_{\mathbf{G}}^{T}$ nonsingular (i.e. $\operatorname{rank}\left(\mathbf{M}_{\mathbf{G}} \cdot\right.$ $\mathbf{M}_{\mathbf{G}}^{T}$ ) $=6$ ) then:

$$
\mathbf{M}_{\mathbf{G}}^{+}=\mathbf{M}_{\mathbf{G}}^{T} \cdot\left(\mathbf{M}_{\mathbf{G}} \cdot \mathbf{M}_{\mathbf{G}}^{T}\right)^{-1}
$$

The dimension of the Jacobian relation $\mathbf{M}_{\mathbf{G}}$ is $(3 \times 4$ (; ( $l \times n$ ( in the general case where: if $n>m$ the system is overactuated, $l$ denoting the size of the configuration space ( 3 in our horizontal case). If the actuator configuration is well posed, the dimension of the kernel of the Jacobian is $(l \times l($. Then $(n-l$ ( added compatible constraints can be considered in the actuation allocation. The task function approach is classicaly used for the control of redundant manipulator, including humanoid systems.

Using this approach, we will complement the jacobian relations with the consideration of an 'operating point' for the actuation system, which avoids dead-zone inputs while providing the actuation demand $\boldsymbol{F}_{\boldsymbol{B}}^{\boldsymbol{d}}$ (when possible), as described in the sequel. Our problem of dead-zone is expressed as an additional constraint, as in [5]. We follow a similar approach and extend this result to dead-zone avoidance.

\section{Thruster identification}

We have performed experiments on a thruster $P_{i}$, measuring the produced thrust $F_{m, i}$ and the current consumption $\left(\mathrm{C}_{m, i}\right.$ ( versus the input $c_{m, i}$. Hence, we have identified the response of Figure 3, where dead-zone effect is clearly shown, and in which the command does not induce any rotation of the propeller, neither current consumption, as shown on Figure 4.

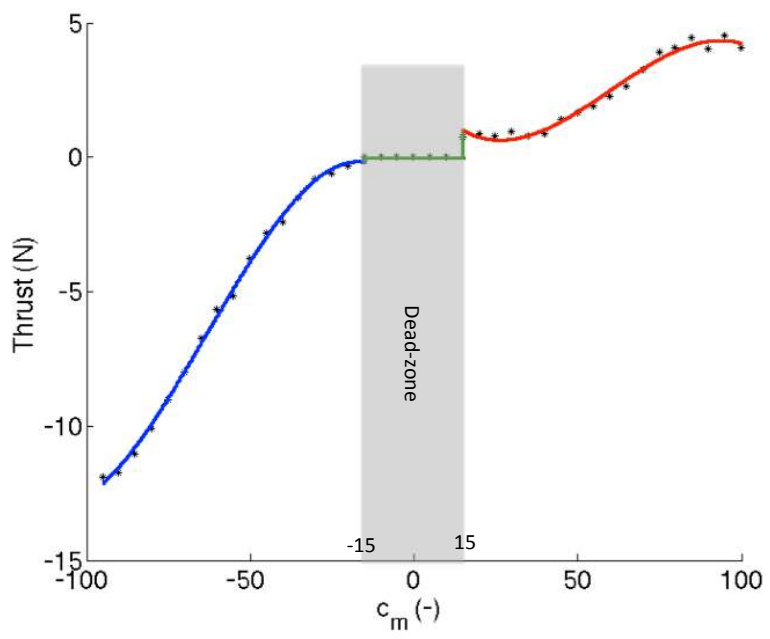

Figure 3. Characteristic of the thrust $F_{m, i}=f\left(c_{m, i}\right)$ 
The experimental response given at Figure 3 is fitted with two third order polynomial expressions, resulting in the expression of the function $F_{m, i}=f\left(c_{m, i}\right)$, and its inverse $c_{m, i}=f^{-1}\left(F_{m, i}\right)$.

$$
F_{m, i}\left(c_{m, i}\right)=\left\{\begin{array}{c}
\sum_{j=0}^{3} a_{j} \cdot c_{m, i}^{j}, \text { if } c_{m, i} \in[-100,-15] \\
\left.0, \text { if } c_{m, i} \in\right]-15,15[ \\
\sum_{j=0}^{3} b_{j} \cdot c_{m, i}^{j}, \text { if } c_{m, i} \in[15,100]
\end{array}\right.
$$

where the identified values of the polynomial coefficients are given at Table 1.

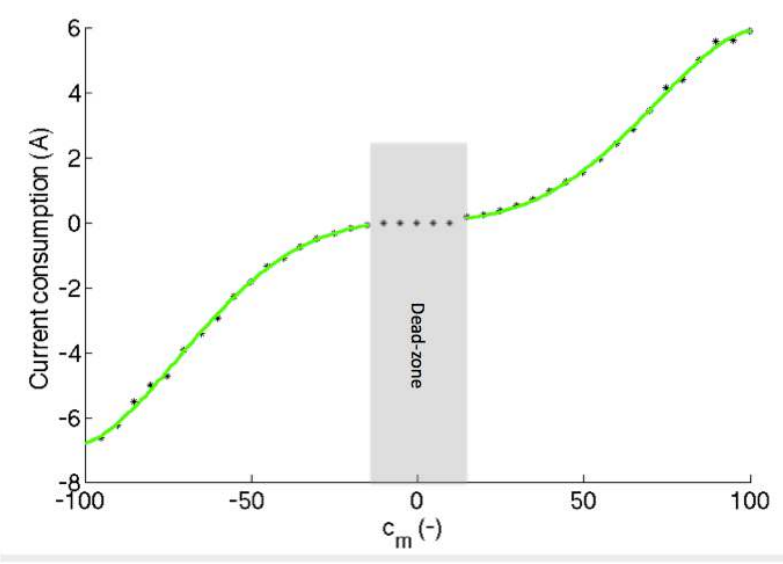

Figure 4. Characteristic of the current consumption $C_{m, i}=f\left(c_{m, i}\right)$

Note that the achievable thrust belongs to: $F_{m, i} \in$ $[-11.8 N,-0.31 N] \cup[0.75 N, 4.51 N]$. Hence, the inverse thrust characteristic is similarly identified as:

$$
c_{m, i}\left(F_{m, i}\right)=\left\{\begin{array}{c}
\sum_{j=0}^{3} d_{j} \cdot F_{m, i}^{j}, \text { if } F_{m, i} \in[-11.8 N,-0.31 N] \\
\left.0, \text { if } F_{m, i} \in\right]-0.31 N, 0.75 N[ \\
\sum_{j=0}^{3} e_{j} \cdot F_{m, i}^{j}, \text { if } F_{m, i} \in[0.75 N, 4.51 N]
\end{array}\right.
$$

where coefficients are given at Table 1.

Figure 5 shows another view of the dead-zone effect, on the overall actuation system, where the 4 thrusters command has been paired $\left(c_{m, 1}=c_{m, 2}=c_{x}\right.$ and $c_{m, 3}=c_{m, 4}=c_{y}$, where these inputs are expressed in terms of PWM). We observe the resulting thrust $F_{u}$. The gray zone corresponds to a response where, at least, one of the thruster's commands fall in the dead-zone.

TABLE I. COEFFICIENTS OF THE POLYNOMIAL CHARACTERISTIC OF THE THRUST PRODUCED BY A SINGLE MOTOR.

\begin{tabular}{|c|c|c|c|c|}
\hline $\boldsymbol{j}$ & $\mathbf{3}$ & $\mathbf{2}$ & $\mathbf{1}$ & $\mathbf{0}$ \\
\hline $\boldsymbol{a}_{\boldsymbol{j}}$ & $-3.2296 \cdot 10^{-5}$ & -0.0062 & -0.1823 & -1.6491 \\
\hline $\boldsymbol{b}_{\boldsymbol{j}}$ & $-2.4378 \cdot 10^{-5}$ & 0.0044 & -0.1812 & 2.8060 \\
\hline $\boldsymbol{d}_{\boldsymbol{j}}$ & 0,063 & 1,28 & 12,8 & $-17,39$ \\
\hline $\boldsymbol{e}_{\boldsymbol{j}}$ & 1,55 & $-14,02$ & 54,34 & $-9,05$ \\
\hline
\end{tabular}

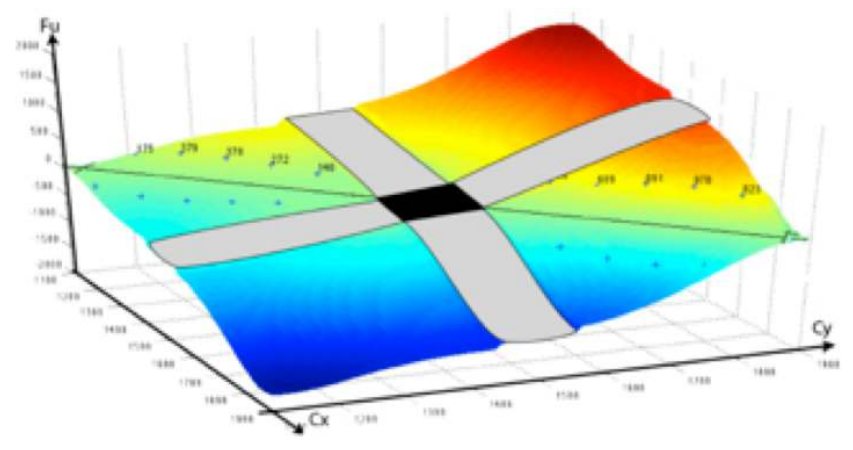

Figure 5. $\quad \boldsymbol{F}_{\boldsymbol{u}}$ in $3 \mathrm{D}$ representation $\left(\boldsymbol{F}_{\boldsymbol{u}}\right.$ vs $\boldsymbol{c}_{\boldsymbol{x}}$ and $\left.\mathrm{c}_{\boldsymbol{y}}\right)$

The usual approach to solve this mechanical problem is to remove from the accessible commands the part corresponding to the dead-zone, as exposed in the next chapter.

\section{DEAD-ZONE EFFECT REMOVAL}

Simply removing from the accessible motor inputs the part corresponding to the dead-zone will induce a modification on the thruster response as depicted in Figure 6, from 6(1) to 6(2). Clearly the thruster is not able to produce low forces, and presents an undesired discontinuity around the origin.

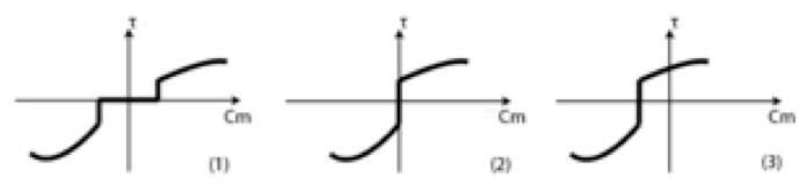

Figure 6. Management of dead zones (1: nominal ; 2 : contraction ; 3 : setting operating point)

Moreover, let's consider the question of heading regulation, where the system is required to maintain a desired yaw angle $\psi_{d}$. Let $\tilde{\psi}=\psi_{d}-\psi$ denote the heading error. Theoretically, a simple proportional control $\Gamma_{r}=K_{r} \cdot \tilde{\psi}$, where $K_{r}$ is an arbitrary positive gain is sufficient. Nevertheless, note that when $\tilde{\psi}$ fluctuates around 0 , the control induces a change of the sign of $\Gamma_{r}$, accordingly. Using the $\mathbf{M}_{\mathbf{G}}$ control allocation matrix will result in motors' inputs that switch around 0 , producing system oscillations around the objective. These oscillations depend on the ability of the thrusters to rapidly invert the propeller rotation, which is poor in our situation, and produces an undesired fatigue of the actuation system.

In order to avoid this situation, we propose to exploit the 'vectorial' configuration of the thrusters, which has the following property:

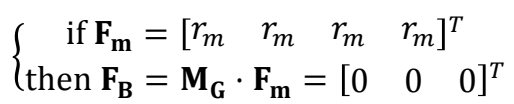

for all accessible $r_{m}$, called motor regime in the sequel, and defining an operating point for the actuation system, as Figure 6(3) illustrates. Doing so, the heading regulation is done far from dead-zone and induces a smooth convergence to 
the objective. The consideration of this offset $r_{m}$ within the control allocation expression results in the following expression:

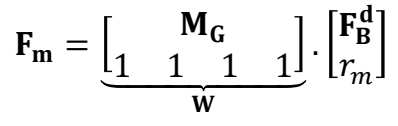

It is quite interesting to note that allocation matrix $\mathbf{W}$ is square and invertible. Its inversion results in the following expression:

$$
\left[\begin{array}{l}
\mathbf{F}_{\mathbf{B}} \\
r_{m}
\end{array}\right]=\underbrace{\left[\begin{array}{r}
1 / 4 \\
\mathbf{M}_{\mathbf{G}}^{+} \\
1 / 4 \\
1 / 4 \\
1 / 4
\end{array}\right]}_{M} \cdot \mathbf{F}_{\mathbf{m}}
$$

The drawback of this approach is, of course, an increase in the actuation activity thus inducing higher energy consumption. Note that placing the $r_{m}$ motor regime in the most vertical part of the thruster's characteristic provides optimal system reactivity, which might be necessary for a short period of time, depending on the mission requirements.

We thus propose the following solution, for the choice of $r_{m}$, which minimizes the impact on power consumption.

\section{A. Optimization}

The principle is to identify an operating point, i.e. the common offset $r_{m}$, in order to provide resulting motors' inputs outside the dead-zone, while respecting saturation constraints. Note that, among all possible $r_{m}$, choosing the smallest one minimizes the effect on energy consumption. The algorithm operates two different transformations on initial, and potentially problematic, motors' inputs $c_{m, i}$. Consider Figure $8(1)$, where $c_{m, i}$ are placed on a saturated axis, and where the dead-zone appears between $\left[c_{d z,-}, c_{d z,+}\right]$. Consider now Figure 8(2). This distribution is clearly not feasible: two inputs exceed limits and a third one lies in the dead-zone. The first operation consists in computing the minimum offset $r_{m}$, Figure 8(3-a), which provides a free dead-zone distribution. The common application of this offset on all inputs can push some of them beyond saturation limits.

The second operation consists in the application of a linear homothetic compression, which preserves the mutual ratios between $c_{m, i}$, as depicted on Figure 7(3-b).

Straightforward analysis of the problem shows that in case of a problematic distribution of $n$ (4 in our case) motors' inputs, there are $n+1$ possible configurations, i.e $n+1$ acceptable $\left(r_{m}, \rho_{m}\right)$.

Note that the application of the compression ratio $\rho_{m}$ modifies the initial actuation demand $\mathbf{F}_{\mathbf{B}}^{\mathbf{d}}$, which is not the case for the application of the offset $r_{m}$. Due to the low complexity of the problem and the small number of solutions, we designed an algorithm, which exhaustively investigate all possible cases, as shown on Figure 7(4). The algorithm reported at Figure 8 describes the process.
(1)
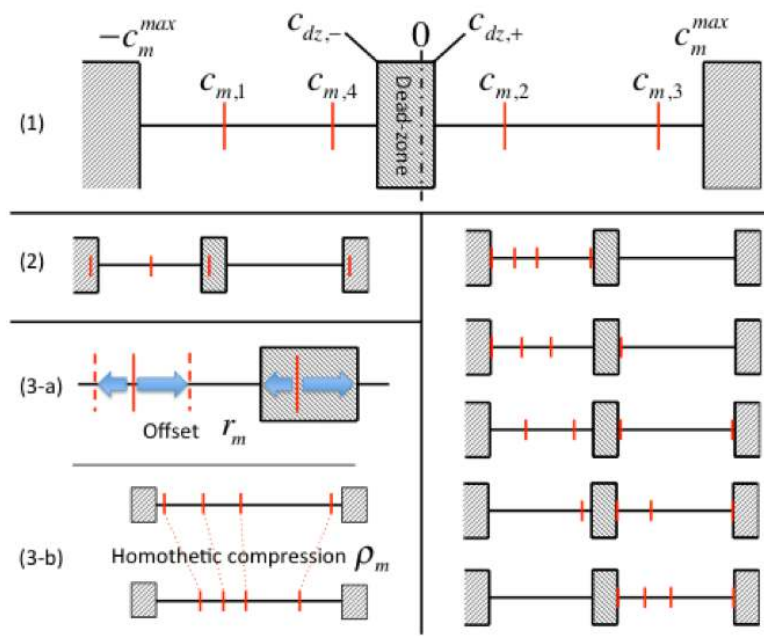

(4)

Figure 7. Example of operating point for optimization

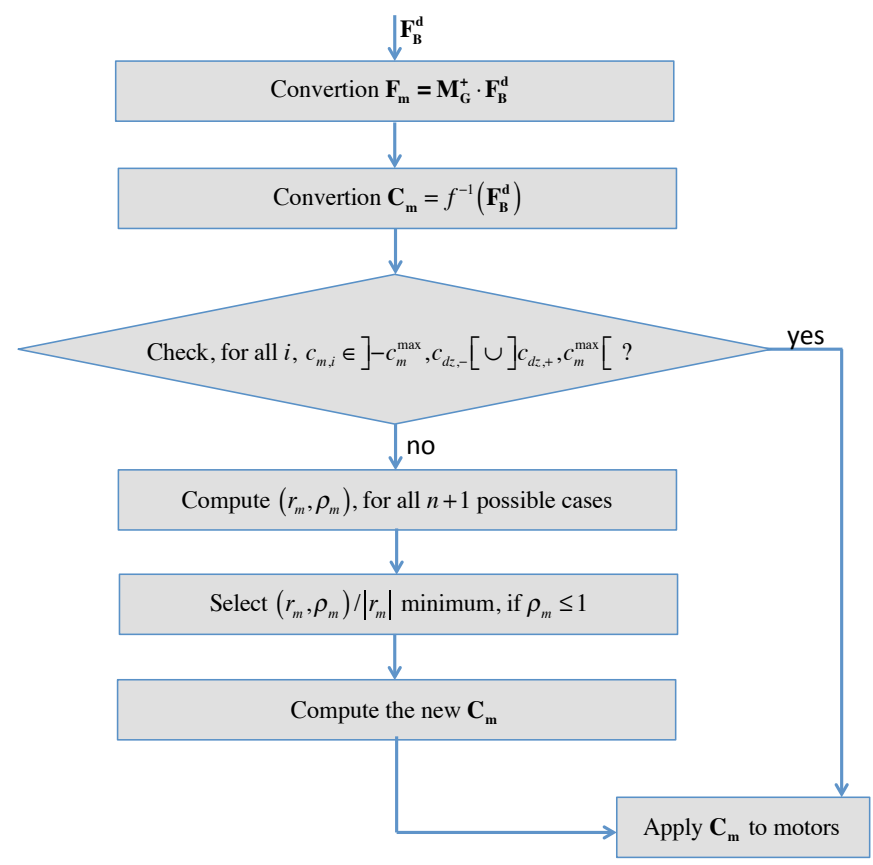

Figure 8. Optimisation algorithm

Consider the actuation demand $\mathbf{F}_{\mathbf{B}}^{\mathbf{d}}$, coming from the operator, or resulting from the computation of a control law. The first two steps convert this demand in terms of motor inputs $\mathbf{c}_{\mathbf{m}}$. A test is then performed to verify if any of the motor inputs lies in the dead-zone, or is beyond actuation limits. In case of a problematic distribution, each of the five situations, described at Figure 8(4), is investigated and couples $\left(r_{m}, \rho_{m}\right)$ are computed. Among these solutions, we reject the ones corresponding to $\rho_{m}>1$. Indeed, in the case where $\rho_{m} \neq 1$, the actuation demand $\mathbf{F}_{\mathbf{B}}^{\mathbf{d}}$ is not respected. Equation 11 summarizes the overall process undergone by the actuation demand.

$$
\mathbf{F}_{\mathbf{B}}=\mathbf{M}_{\mathbf{G}}^{+} \cdot f\left(\rho_{m} \cdot f^{-1}\left(\mathbf{w} \cdot\left[\begin{array}{c}
\mathbf{F}_{\mathbf{B}}^{\mathbf{d}} \\
r_{m}
\end{array}\right]\right)\right)
$$


It is clear that if $\rho_{m}>1$ the resulting forces and torque produced by the actuation system $\left(\mathbf{F}_{\mathbf{B}}\right)$ will be bigger than $\mathbf{F}_{\mathbf{B}}^{\mathbf{d}}$, thus questioning the operator, or control law, reactivity capacity. Then we just allow $\rho_{m} \leq 1$.

The following section gives experimental results obtained when applying the proposed dispatcher-based approach on the Jack robot.

\section{EXPERIMENTATIONS}

The Figure 9 shows the experimentations realized on the Jack system in a pool.

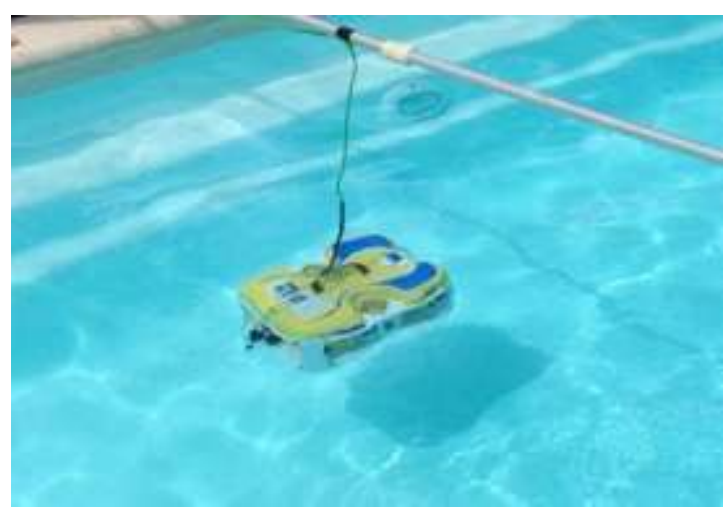

Figure 9. Picture of experimentation.

In order to illustrate the performances of our solution, we use a basic PD yaw controller:

$$
\Gamma_{r}=K_{P} \cdot\left(\psi_{d}-\psi\right)-K_{D} \cdot \dot{\psi}
$$

where $\psi_{d}$ is the desired heading and $K_{P}=50$ and $K_{D}=1$ are positive gains. The control output is then sent to the different dispatchers (static, compressed or dynamic) during three experiments, reported at Figure 11. The desired heading reference has been chosen to $\psi_{D}=120^{\circ}$. Initial position has been roughly set to $20^{\circ}$. At approximately $28 \mathrm{~s}$, the control is disengaged and the robot is manually oriented at $200^{\circ}$, thus simulating a similar external perturbation. Then the control is engaged again.

The first experiment considers the static dispatcher (eq. 4) with the basic motor characteristic reported in 6(1). Results are drawn in blue.

The second experiment considers the same dispatcher, but removes the dead-zone (compressed) from the accessible motor inputs, as shown in 6(2). Results are in red.

Lastly, the third experiment considers the use of the dynamic dispatcher, which follows the placement algorithm described previously. Results are given in black.
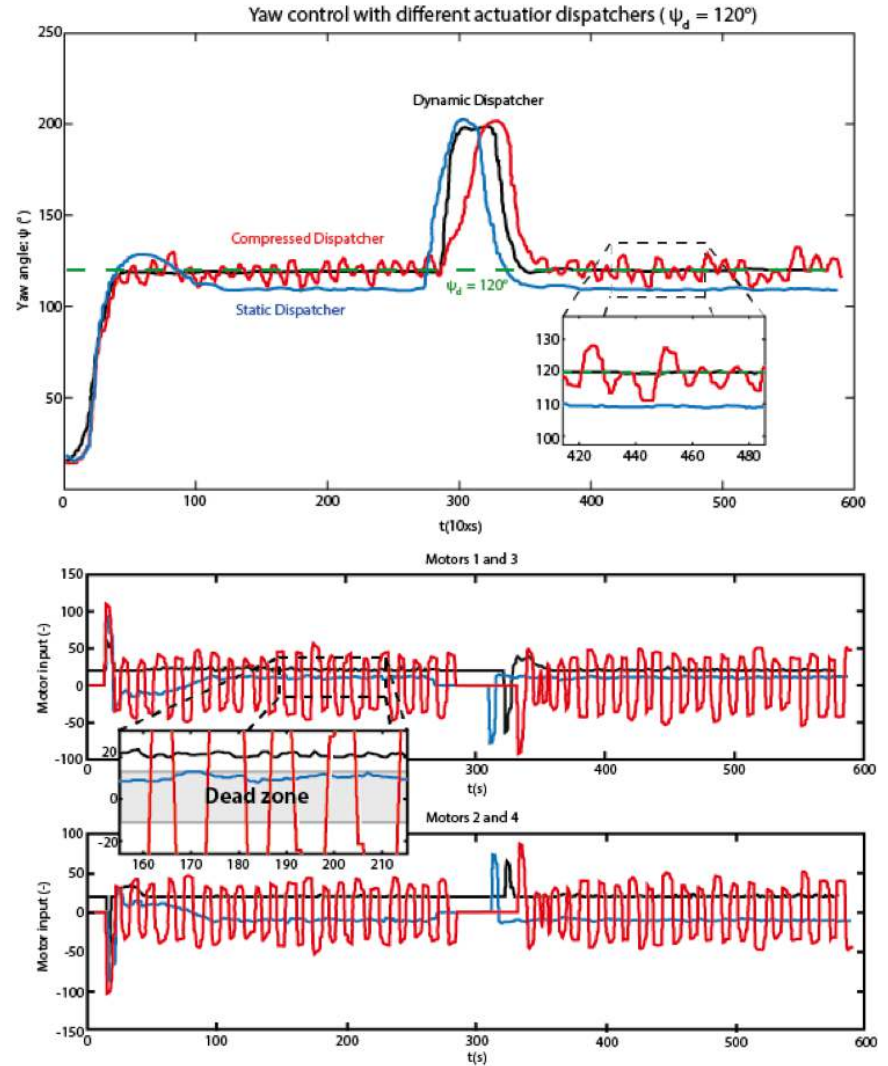

Figure 10. Experimental results

\section{A. Analysis of the static dispatcher response}

The blue curves on Figure 11 clearly show the limitations of the static dispatcher. Indeed, the control law provides control inputs regardless to the presence of the dead-zone. The motor characteristic reported on Figure 3 shows a dead-zone between $c_{m, d z} \in[-15,15]$. Considering the control law (12), given $K_{P}=50$, the expected static error is $\tilde{\psi}_{\text {stat }}=\frac{15}{50}=$ $0.3 \mathrm{rad} \approx 17.18^{\circ}$. This corresponds to a heading error that won't induce any thrusters' reaction, as one can see on experimental results of Figure 11. Note that this static error is the result of a default of the actuation system, which is not able to produce low thrust. Hence the consideration of an integral gain in control 12 will not compensate this static error.

\section{B. Analysis of the compressed dispatcher response}

The red curve on Figure 11 shows the performances of the compressed dispatcher. This one is similar to the previous one, but refers to a motor response where the dead-zone has been contracted (Figure 6(2)), bounding the dead-zone with admissible values of the motor inputs such that $c_{m} \in$ $[-100,-20] \cup[20,100]$, covering the real dead-zone $\left(c_{m, d z} \in[-15,15]\right)$. The result indicates that the static error is compensated but the system oscillates around the desired value. These oscillations are induced by the time of reaction of the thrusters to produce an inverted thrust. A decrease of the gain $K_{P}$ will reduce the amplitude of these oscillations, but 
won't avoid them. This situation, of course, induces an unnecessary fatigue of the motors.

\section{Analysis of the dynamic dispatcher response}

This time, the input for each motor is computed according to the algorithm previously described. A common motor regime is computed in order to place the operating point of the motors outside the dead-zones. If the control output is over 100 (saturation case), the compression coefficient is applied. The application of this algorithm clearly improves the control performances, since the oscillations and the static error no longer exist.

The impact on energy consumption can be estimated by analyzing the evolution of the actuator activity, through the curves of Figure 11, where the evolution of the input of motor 1 and 3 are drawn. Since the use of the first dispatcher induces a huge static error, we only compare the motors' inputs activity of the second and third solutions. We compute the following energy-like criterion for both actuation responses between instants $t_{\text {init }}=1.5 \mathrm{~s}$ and $t_{\text {end }}=25 \mathrm{~s}$.

$$
\begin{aligned}
& E_{\text {Comp_Dispatcher }}=\int_{1.5}^{25} c_{m, 1} \cdot \mathrm{d} t=863.6 \\
& E_{\text {Adapt_Dispatcher }}=\int_{1.5}^{25} c_{m, 1} \cdot \mathrm{d} t=526.9
\end{aligned}
$$

Hence, we conclude, as expected, that despite the offset $r_{m}$ present on the response of the adaptive dispatcher, the energy consumption is smaller than the one induced by the oscillatory behavior of the compressed dispatcher.

\section{CONCLUSION AND FUTURE WORK}

The optimization of thrusters' allocation using our method allows removing the effect of the dead-zone, while respecting the desired actuation demand. The motors saturation effect is avoided using a homothetic compression of the motor inputs. Moreover, this method allows for choosing an 'operating point' for the actuation system, hence providing the management of the system reactivity. The effect of the method on the energy consumption is considered. Experimental tests validate the approach.

The next step will be to increase the system manoeuvrability by adding a similar actuation system, resulting in a hyper-redundant system carrying 12 thrusters. Following the same approach, this system will allows for concurrently considering added secondary tasks in the control objectives.

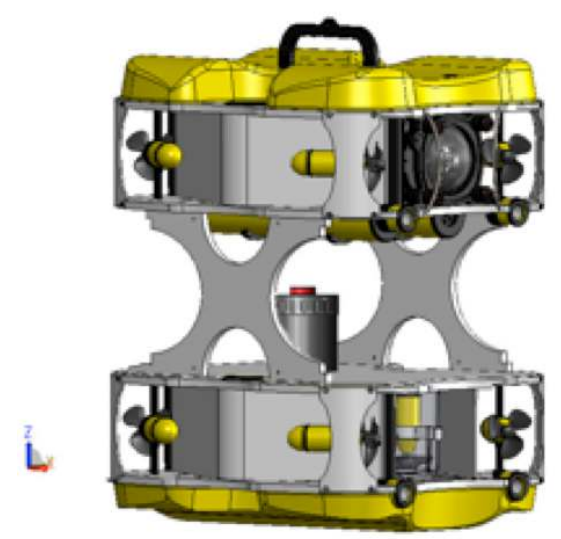

Figure 12. The future hyper-redundant Jack system.

\section{ACKNOWLEDGMENT}

The authors would like to warmly thank S. Louis for his constant help on this study and the French labex NUMEV for supporting this research.

\section{REFERENCES}

[1] E. Omerdic and G. Roberts. "Thruster fault diagnosis and accommodation for open-frame underwater vehicle". Control engineering practice, Vol. 12, pp. 1575-1598, 2004.

[2] J. Garus."Fault tolerant control of remotely operated vehicle". IEEE int. Conference on methods and models in automation and robotics, pp. 217-221, Miedzyzdroje, 2003, Poland.

[3] T. I. Fossen. "Guidance and control of ocean vehicles", John Wiley and Sons Ltd., 1994, Chichester.

[4] J. Garus. "Optimization of thrust allocation in the propulsion system of an underwater vehicule. Applied mathematic and computer science”, Vol. 14, No. 4, pp. 461-467, 2004.

[5] G. Indiveri and G. Parlangeli. "On thruster allocation, fault detection and accommodation issues for underwater robotic vehicles". IEEE ISCCSP 2006 Second International Symposium on Communications, Control, and Signal Processing, March 13-15, 2006, Marrakech, Morocco.

[6] C. Samson, B. Espiau and M. Le Borgne. "Robot Control: The Task Function Approach", Oxford University Press, 1991,UK.

[7] A. Hanai, K. Rosa, S.K Choi and J. Yuh. "Experimental Analysis and Implementation of Redundant Thrusters for Underwater Robots". Proceedings of 2004 IEEE/RSJ International Conference on Intelligent Robots and Systems, pp.1109-1114, September 28 - October 2, 2004, Sendai, Japan

[8] T. Haberkorn, M. Chyba, R. Smith, S.K Choi, G. Marani and C. Mcleod. "Efficient control of an autonomous underwater vehicle while accounting for thruster failure", Ocean Engineering, Vol. 35, 1, pp. 6376, January 2008.

[9] F. Flacco. "Motion control of redundant robot under joint constraints: Saturation in the null space". IEEE International Conference on Robotics and Automation (ICRA), pp. 285-292, May 14-18, 2012, Saint Paul,MN, USA.

[10] Y. Nakamura, H. Hanafusa and T. Yoshikawa. "Task-Priority Based Redundancy Control of Robot Manipulators" In the International Journal of Robotic Research, 1987 6:3. DOI: $10.1177 / 027836498700600201$ 